\title{
ANDES

\section{Diagnóstico de Reflujo faringolaríngeo mediante Impedanciometría con pHmetría esofágica en niños con disfonía crónica}

\author{
Diagnosis of laryngopharyngeal Reflux by Impedance with esophageal pHmetry in \\ children with chronic dysphonia
}

\author{
Elisa A. Aranda S. ${ }^{a}$, Teresa Alarcón O. ${ }^{\mathrm{b}}$, Rossana Faúndez H. ${ }^{\mathrm{b}}$, Margarita Arancibia S. ${ }^{\mathrm{c}}$
}

aBecada. Programa de Subespecialidad en Gastroenterología Pediátrica, Facultad de Medicina, Universidad de Chile. Santiago, Chile bServicio de Pediatría, Hospital San Juan de Dios, Universidad de Chile. Santiago, Chile

'Servicio de Otorrinolaringología, Hospital San Juan de Dios, Universidad de Chile. Santiago, Chile

Recibido: 12 de agosto de 2020; Aceptado: 7 de mayo de 2021

¿Qué se sabe del tema que trata este estudio?

Reflujo faringolaríngeo es una de las condiciones inflamatorias crónicas de la laringe más frecuentemente descritas. Pero la mayoría de estos pacientes no muestran síntomas clásicos de enfermedad por reflujo gastroesofágico, lo que dificulta realizar o descartar este diagnóstico sólo con la clínica.

\section{¿Qué aporta este estudio a lo ya conocido?}

Es el primer reporte nacional pediátrico en niños con disfonía crónica, que utilizando la Impedanciometría con pHmetría esofágica como herramienta diagnóstica permite confirmar con mayor certeza una relación causal entre hallazgos laringoscópicos sospechosos y reflujo faringolaríngeo.

\section{Resumen}

Reflujo Faringolaríngeo (RFL) es el flujo retrógrado del contenido gástrico o duodenal hacia faringe y laringe, generando inflamación en el tracto aerodigestivo superior. Tradicionalmente se utilizaba pHmetría con índice de reflujo ácido. La impedanciometría con pHmetría esofágica (pH-IIM) permite confirmar una relación causal entre síntomas sospechosos y RFL. Objetivos: Estudiar la presencia de RFL diagnosticado por pH-IIM en población pediátrica consultante por disfonía crónica y hallazgos laringoscópicos sugerentes de RFL, además, evaluar la concordancia entre pH-IIM y pHmetría tradicional. Pacientes y Método: Estudio descriptivo, prospectivo de pacientes consultantes en policlínico de Gastroenterología u Otorrinolaringología por disfonía crónica, cuya nasofibrolaringoscopía (NFL) fue sugerente de RFL. Los pacientes fueron hospitalizados para realizar pH-IIM de $24 \mathrm{hrs}$. Se excluyeron pacientes con antecedentes mórbidos congénitos o adquiridos. Se consideró RFL patológico si había 3 o más episodios de reflujo ácido a nivel proximal en pH-IIM. Se evaluó frecuencia de $\mathrm{pHmetría} \mathrm{tradicional} \mathrm{y} \mathrm{pH}$-IIM alteradas y la concordancia entre ambos métodos. Resultados: Se reclutaron 12 pacientes, 10 varones, de 6 a 15 años. En 9/12 se confirmó RFL patológico por pH-IIM,
Palabras clave:

Reflujo

Faringolaríngeo;

Impedanciometría con pHmetría Esofágica;

Índice de Reflujo

Ácido;

Nasofibrolaringoscopía

Correspondencia:

Elisa A. Aranda S

andimed75@gmail.com 
de los cuales $2 / 9$ presentaban pHmetría tradicional en rangos normales y 7/9 pHmetría alterada. En 3 pacientes se descartó RFL por pH-IIM proximal normal. La concordancia entre pH-IIM y pHmetría tradicional fue aceptable (kappa 0,4). Conclusiones: 75\% de los pacientes con disfonía y NFL sugerente mostraron evidencia objetiva de RFL patológico. Dado que sólo con la evaluación clínica, NFL y pHmetría convencional no es posible hacer diagnóstico de RFL, recomendamos hacer pH-IIM para mayor certeza diagnóstica, evitando un tratamiento innecesario y no libre de efectos indeseados en $25 \%$ de los casos.

\section{Abstract}

Laryngopharyngeal Reflux (LPR) is the retrograde flow of gastric or duodenal contents into the pharynx and larynx, causing inflammation in the upper aerodigestive tract. Traditionally, a $\mathrm{pH}$ monitoring study with an acid reflux index was used. The use of multichannel intraluminal impedance testing with $\mathrm{pH}$ monitoring $(\mathrm{MII}-\mathrm{pH})$ confirms a causal relationship between suspicious symptoms and LPR. Objectives: To evaluate LPR diagnosed by MII-pH in the pediatric population consulting due to chronic dysphonia and laryngoscopic findings suggestive of LPR, in addition, to measure the concordance between MII-pH and traditional pH monitoring. Patients and Method: Descriptive, prospective study of patients consulting at the Gastroenterology or Otorhinolaryngology polyclinic due to chronic dysphonia, whose nasofibrolaryngoscopy (NFL) was suggestive of LPR. The patients were hospitalized for a 24-hour MII-pH. Patients with a congenital or acquired morbid history were excluded. Pathological LPR was considered if there were 3 or more acid reflux episodes at the proximal level in MII-pH. The frequency of traditional $\mathrm{pH}$ monitoring and altered MII-pH and the concordance between both methods were evaluated. Results: 12 patients were recruited, 10 men, 6 to 15 years old. On 9/12, pathological LPR was confirmed by MII-pH, of which 2/9 had traditional pH measurements in normal ranges and 7/9 altered $\mathrm{pH}$ measurements. In 3 patients, LPR was ruled out by normal proximal MII-pH. The concordance between MII-pH and traditional $\mathrm{pH}$ monitoring was acceptable (kappa 0.4 ). Conclusions: $75 \%$ of the patients with dysphonia and suggestive NFL showed objective evidence of pathological LPR. Since only with the clinical evaluation, NFL and conventional $\mathrm{pH}$ monitoring it is not possible to diagnose LPR, we recommend perform MII-pH for greater diagnostic certainty, avoiding unnecessary treatment, and with unwanted effects in $25 \%$ of cases.
Keywords:

Laryngopharyngeal

Reflux;

Impedance with

Esophageal $\mathrm{pH}$

Measurement;

Acid Reflux Index;

Nasofibrolaryngoscopy

\section{Introduccion}

El Reflujo Faringolaríngeo (RFL) se define como flujo retrógrado del contenido gástrico o duodenal hacia la faringe y laringe dando lugar a irritación e inflamación en el tracto aerodigestivo superior ${ }^{1,2,3}$. RFL pediátrico tiende a considerarse una condición patológica única y distinta al reflujo gastroesofágico (RGE), pero continúa siendo una entidad con más preguntas que respuestas ${ }^{2,4}$.

La literatura otorrinolaringológica describe la existencia de reflujo gástrico con manifestaciones extradigestivas laríngeas desde hace al menos cuatro décadas, pero la conceptualización actual en un enfoque pediátrico-gastroenterológico fue descrita por James Koufman como RFL recién a principio de los noventa ${ }^{5}$. Entidad cada vez más conocida, con publicaciones que intentan explicar el trastorno fisiopatológico ${ }^{1}$.

El RFL es una de las condiciones inflamatorias crónicas de la laringe más frecuentes encontradas, afecta al $8-20 \%$ de la población general, $4-10 \%$ de pacientes de la consulta con otorrinolaringólogo, $1 \%$ de pacientes de atención primaria y hasta el $75 \%$ de pacientes con síntomas otorrinolaringológicos refractarios. RFL puede afectar al 50-78\% de población con molestias de voz y $91 \%$ de casos de desórdenes de la voz en ancianos ${ }^{6}$. La prevalencia de RFL en niños sigue siendo desconocida, existen pocos datos al respecto ${ }^{2}$.

La etiología es multifactorial, incluyendo disfunción del esfínter esofágico superior, el tiempo de exposición y nivel de sensibilidad del tejido ${ }^{1,7}$. Se ha descrito mayor sensibilidad al reflujo del epitelio laríngeo que del esofágico. Sólo se necesitarían 3 episodios de RFL, con $\mathrm{pH}$ inferior a 4 a la semana, para que se produzca el daño a nivel laríngeo, a diferencia del RGE que requiere aproximadamente 50 episodios semanales para producir algún grado de daño esofágico. La explicación de esta mayor sensibilidad epitelial permanece aún poco clara, pero se cree que la presencia de un $\mathrm{pH}$ bajo a nivel de la laringe, acompañado de la presencia de pepsina, generaría una reacción, que aumentaría la producción de proteínas de estrés, afectando la reparación epitelial local, provocando daño ${ }^{1}$. 
Se han descrito estudios de inmunohistoquímica en que se encuentran concentraciones de pepsina y depleción de isoenzima III de anhidrasa carbónica en biopsia laríngea de casos documentados de $\mathrm{RFL}^{8}$. Además, debemos agregar la participación de enzimas pancreáticas en el reflujo duodenal ${ }^{6}$.

La irritación directa por reflujo puede causar tos y asfixia (laringoespasmo) debido a que la sensibilidad en las terminaciones sensitivas laríngeas está regulada por inflamación local ${ }^{8}$. Esta combinación de factores puede conducir a edema del pliegue vocal, úlceras por contacto y granulomas que causan otros síntomas asociados a RFL, como son disfonía, globus faríngeo y odinofagia ${ }^{8}$. En cuanto a la disfonía, ésta implica cambios anatopatológicos en el epitelio que se asocian a otros factores que pueden interactuar.

La mayoría de pacientes con RFL no muestran síntomas clásicos de enfermedad por RGE por lo cual es difícil realizar su diagnóstico. Además, sus síntomas son inespecíficos y el diagnóstico potencialmente confuso.

La mayoría de los signos laringoscópicos considerados sugerentes de RFL tienen pobre especificidad, ya que pueden ser encontrados hasta en el $70 \%$ de sujetos asintomáticos?.

Aunque no existe una prueba diagnóstica ideal para RFL debido a algunas limitaciones, se ha desarrollado la Impedanciometría Intraluminal Multicanal de 24 hrs. (IIM), que es una técnica de monitoreo de RGE que permite evaluar el movimiento de líquidos, sólidos y aire, independiente del $\mathrm{pH}$ del material refluido (ácido/débilmente ácido/no ácido) ${ }^{10}$. Por otro lado la pHmetría esofágica $(\mathrm{pH})$ de 24 hrs. evalúa sólo reflujo ácido ${ }^{11}$.

La pH-IIM (combinación de 2 técnicas anteriores) está disponible con sondas pediátricas desde el año 2002. La Sociedad Norteamericana de Gastroenterología Pediátrica, Hepatología y Nutrición (NASPGHAN) y Sociedad Europea de Gastroenterología Pediátrica, Hepatología y Nutrición (ESPGHAN) refieren que la "pH-IIM, es superior a la monitorización de $\mathrm{pH}$ por sí sola para la evaluación de la relación temporal entre síntomas y RGE"12,13.

Antes del uso de la pH-IIM esofágica de 24 horas no existía forma de evidenciar una relación causal entre síntomas sospechosos como tos crónica sin causa conocida, disfonía o laringitis recurrente y RFL. La asociación de esta herramienta diagnóstica frente a hallazgos laringoscópicos sugerentes de RFL, pretende aumentar la certeza diagnóstica, disminuir el sobrediagnóstico de RFL y ofrecer un tratamiento más adecuado.

El objetivo de esta investigación fue estudiar la presencia de RFL diagnosticado por pH-IIM en población pediátrica consultante por disfonía crónica y hallazgos laringoscópicos sugerentes de RFL, además, evaluar la concordancia entre pH-IIM y la pHmetría tradicional.

\section{Pacientes y Método}

Estudio descriptivo y prospectivo de pacientes pediátricos que consultaron en policlínico de Gastroenterología u Otorrinolaringología del Hospital San Juan de Dios de Santiago de Chile, entre noviembre 2015 y noviembre del 2016, con disfonía crónica y cuya Nasofibrolaringoscopía (NFL) resultó sugerente de RFL.

Se incluyeron pacientes con antecedente de disfonía y que a la NFL realizado por otorrinolaringólogo hubiera informado algunos de los siguientes hallazgos: eritema de aritenoides, edema laríngeo, granulomas, pseudosulcus o sulcus laríngeo, eritema de comisura posterior, pared posterior de la faringe con aspecto de empedrado, nódulos de cuerdas vocales.

Se excluyeron los pacientes con malformaciones congénitas, cardíacas y digestivas y oro-faciales; historia de cirugía toráxica o abdominal (incluyendo cirugía esofágica o fundoplicatura); fibrosis quística; enfermedad neuromuscular; convulsiones; discapacidad intelectual (por el riesgo de retiro de sonda de pH-IIM durante hospitalización); parálisis cerebral.

\section{Procedimiento}

Los pacientes fueron hospitalizados para realizar estudio con pH-IIM de 24 hrs. En aquellos que estaban con tratamiento con inhibidores de bomba de protones se le indicó suspenderlo una semana antes.

En todos los pacientes se completó un registro de 24 hrs., con equipo Digitrapper pH-Z (24-Hour pH \& Impedance Recorder) con Sensor de impedanciometría y de $\mathrm{pH}$.

Este estudio se realizó instalando a través de una fosa nasal una pequeña sonda en el esófago que registra, juntamente con los cambios de $\mathrm{pH}$, los cambios de la impedancia eléctrica entre dos electrodos contiguos durante el pasaje del bolo alimentario a través del esófago, independientemente de que el contenido sea ácido o no ácido. La dirección del movimiento del bolo puede ser visualizada por la presencia de múltiples electrodos ubicados secuencialmente en un catéter, pudiendo establecerse también la altura a la que llegan los episodios de reflujo ${ }^{14}$.

Previo a instalación se calibró el equipo con 2 soluciones de $\mathrm{pH}$ diferentes ( $\mathrm{pH} 7,0$ y $\mathrm{pH} 4,0)$. Se introdujo sensor trans-nasal humedecido previamente con agua destilada para facilitar el paso a través de las fosas nasales; no se utilizó gel debido a que su presencia en la sonda puede disminuir su exactitud durante el examen $^{12}$. Con radiología simple de tórax y abdomen se comprobó posición de sensor de $\mathrm{pH}$ de la sonda a la al- 
tura de cuerpo de 2 da vértebra por sobre el diafragma.

Los pacientes recibieron fórmulas lácteas habituales y alimentación adecuada a su edad y desarrollo. Los registros fueron realizados en decúbito supino mientras duerme y en posición vertical (sentado, deambula) en vigilia. La idea fue reproducir en lo posible la actividad normal del hogar. Antes del inicio del protocolo, los padres de potenciales sujetos del estudio fueron contactados, se les explicó en qué consistía y los que aceptaron firmaron el consentimiento informado en el que además quedó estipulado la utilización de los datos obtenidos y la publicación de ellos. Pacientes $>$ de 8 años firmaron asentimiento. (Universidad de Chile - Facultad de Medicina, Comité de Ética de Investigación en Seres, Proyecto N¹22-2016, Archivo Acta $\left.\mathrm{n}^{\circ} 1\right)$.

\section{Parámetros considerados}

pHmetría con índice de reflujo (IR) y Puntuación de DeMeester: IR es definido como el porcentaje de tiempo total del registro en que el $\mathrm{pH}$ esofágico es $<4$ para determinar la enfermedad por RGE patológica y refleja la exposición acumulativa del esófago al ácido ${ }^{15}$. Estudios describen que el IR medido por pHmetría tiene una sensibilidad y especificidad 50\% y $82 \%$, respectivamente ${ }^{16}$.

Puntuación de DeMeester habitualmente utilizada en población adulta, considera como exposición ácida anormal una puntuación global de DeMeester $>14,72$ en el esófago distal. Este índice de puntuación combinada se obtiene mediante la suma del cálculo individualizado de cada uno de los seis componentes que lo conforman: número de episodios de reflujo en 24 horas, número de episodios de reflujo más largos de 5 minutos en 24 horas, episodio más largo (minutos), $\%$ de tiempo total con $\mathrm{pH}<4,0$, \% de tiempo con $\mathrm{pH}<4,0$ en bipedestación, \% de tiempo con $\mathrm{pH}<4,0$ supino $^{17}$.

Se consideró pHmetría normal o alterada según índice de reflujo (IR) (normal $<0=7$ ) y puntuación de DeMeester (normal $<\mathrm{o}=14,72$ p95 en adultos), siendo el IR el parámetro más importante.

pH-IIM: Se consideró RGE proximal presente si la caída en la impedancio alcanzó a 1 o a 2 de los sensores más proximales de la sonda de impedancio, catalogándolos de ácidos, débilmente ácidos o no ácidos según registro de sensor de $\mathrm{pH}$ distal. Definimos (de acuerdo con literatura revisada, que sugiere que 3 reflujos proximales de $\mathrm{pH}<\mathrm{a} 4$ a la semana son suficientes para ocasionar daño de epitelio laríngeo) como pH-IIM proximal alterada la presencia de 3 o más episodios de reflujo ácido a nivel proximal por $\mathrm{pH}-\mathrm{IIM}$.

\section{Análisis estadístico}

Se analizó la frecuencia de pHmetría alterada y de pH-IIM alterada en la población estudiada.
Se usaron estadísticas descriptivas, mediana y rango intercuartílico. Los datos fueron analizados en planilla Microsoft excel versión 2012.

Se evaluó la concordancia de la pHmetría tradicional versus la pH-IIM para el diagnóstico de RFL, a través de índice Kappa de Cohen ${ }^{18}$.

\section{Resultados}

Se reclutaron 12 pacientes, 10 de sexo masculino, con una mediana de edad de 10 años y rango intercuartílico de 4 (rango 6 a 15 años).

El presentar pHmetría normal por IR y por Puntuación de DeMeester (4/12), no descartó RFL, ya que la mitad de estos pacientes (2/4) tuvo reflujo proximal, el cual no podría ser detectado si no se complementa este examen con IIM. Por otro lado, de los pacientes que presentaron $\mathrm{pH}$ alterada (8/12), 7/8 presentaron $\mathrm{pH}$-IIM proximal alterada y $1 / 8 \mathrm{pH}$-IIM proximal normal, luego el tener pHmetría alterada no equivale a presencia de RFL.

Es decir, en 4 de los 12 pacientes se habría descartado el RFL por pHmetría normal por IR ácido y por Puntuación de DeMeester, 2 de ellos habrían sido falsos negativos y 2 verdaderos negativos (figura 1 ).

Mirado de otro modo, en 9/12 se documentó reflujo proximal por $\mathrm{pH}$-IIM proximal alterada, de los cuales $2 / 9$ presentaban $\mathrm{pHmetría} \mathrm{por} \mathrm{IR} \mathrm{y} \mathrm{por} \mathrm{Puntuación}$ de DeMeester dentro de rangos normales y 7/9 pHmetría anormal (figura 1).

La concordancia entre pHmetría tradicional y la pH-IIM fue aceptable, Kappa 0,4 (tabla 1).

Los hallazgos más frecuentemente encontrados en NFL tanto en el total de pacientes como en los que presentaron $\mathrm{pH}$-IIM proximal alterada fueron edema o eritema de aritenoides y de comisura posterior (tabla 2).

\section{Discusión}

En el presente estudio se demostró que sólo el 75\% de los pacientes con disfonía y NFL sugerente de RFL muestran evidencia objetiva de RFL patológico, es decir, pH-IIM proximal alterada, en coincidencia con los estudios de la Dra. Rosen y cols. que concluyeron que cuando se combina $\mathrm{pH}-\mathrm{IIM}$, la toma de decisiones clínicas se modifica en aproximadamente $25 \%$ de los pacientes respecto a la clínica y $\mathrm{pHmetría} \mathrm{tradicional}{ }^{19}$.

$\mathrm{Al}$ realizar sólo pHmetría no se habrían diagnosticado 2 pacientes con RFL que fueron rescatados con pH-IIM proximal y se hubiera sobre diagnosticado 1 paciente. Considerar sólo el resultado de los hallazgos laringoscópicos para un tratamiento con inhibidores 


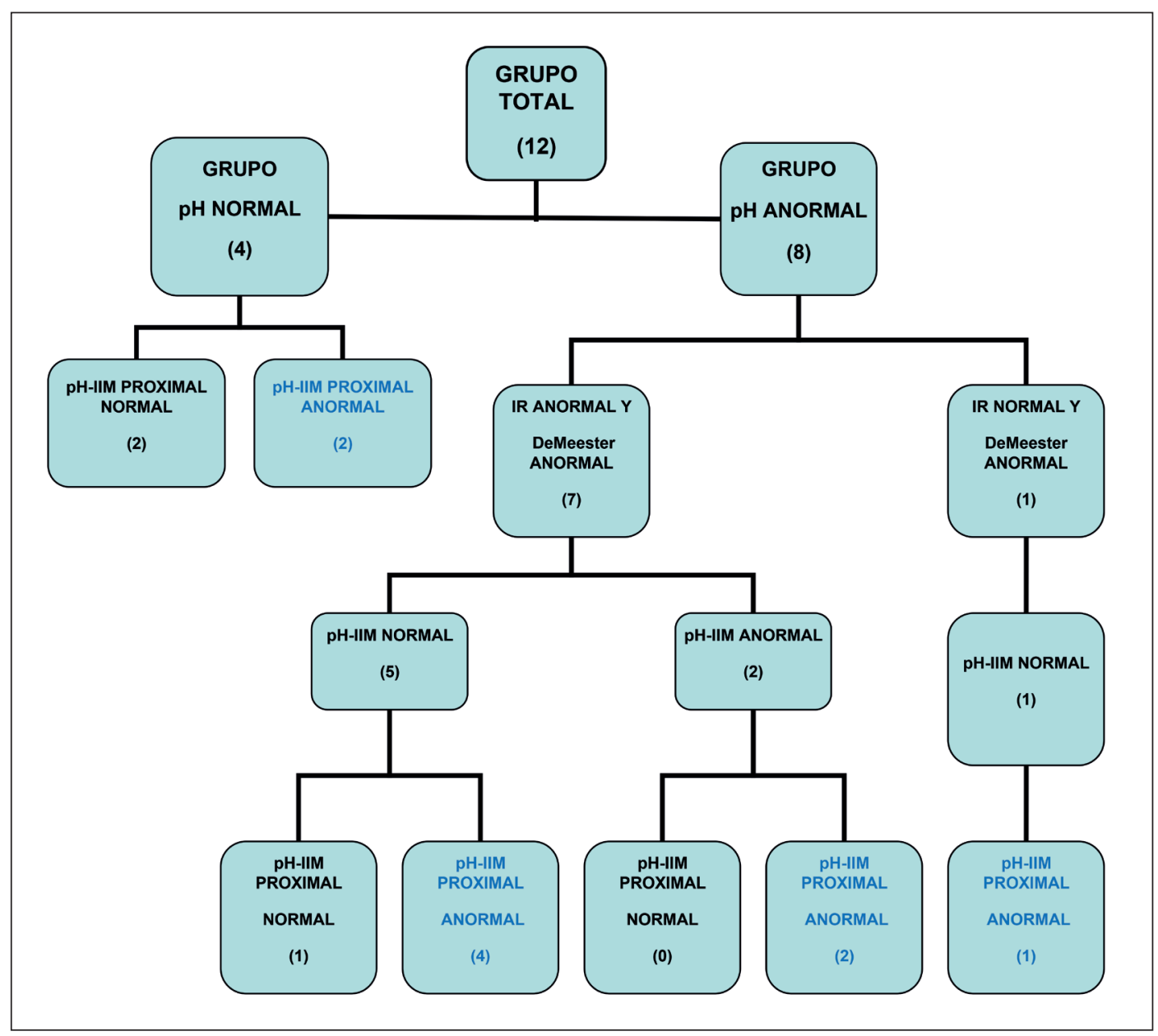

Figura 1. Distribución de pacientes según $\mathrm{pH}$ metría. $\mathrm{pH}=$ pHmetría; $\mathrm{IR}=$ Indice de reflujo ácido (normal $<0=7$ ); Puntuación de DeMeester $=$ Normal $<0=14,72$ (p95); $\mathrm{pH}-\mathrm{IIM}=$ Impedanciometría con pHmetría esofágica.

Tabla 1. Tabla comparativa entre pHmetría convencional e Impedanciometría con pHmetría esofágica

\begin{tabular}{lccc}
\hline & $\begin{array}{c}\mathrm{pH}-\mathrm{IIM} \\
\text { Proximal Alterada }\end{array}$ & $\begin{array}{c}\mathrm{pH}-\mathrm{IIM} \\
\text { Proximal Normal }\end{array}$ & Total \\
\hline IR ácido y/o Puntuación de DeMeester Alterada & 7 & 1 & 8 \\
IR ácido y/o Puntuación de DeMeester Normal & 2 & 2 & 4 \\
Total & 9 & 3 & 12 \\
\hline
\end{tabular}

Kappa = 0,4. IR = Índice de Reflujo Ácido; pH-IIM = Impedanciometría con pHmetría esofágica.

Tabla 2. Hallazgos de nasofibrolaringoscopía

\begin{tabular}{lccc}
\hline Hallazgos NFL & Pacientes con $\mathrm{pH}-\mathrm{IIM}$ proximal alterada & Pacientes con pH-IIM proximal normal \\
& $(9)$ & $(3)$ & 1 \\
\hline Edema o eritema de aritenoides & 8 & 2 \\
Edema o eritema de comisura posterior & 7 & 2 & 1 \\
Pared posterior de faringe en empedrado & 4 & 3 \\
Nódulos de cuerdas vocales o esbozo de ellos & 2 & \\
\hline
\end{tabular}

$\mathrm{NFL}=$ Nasofibrolaringoscopía; $\mathrm{pH}-\mathrm{IIM}=$ Impedanciometría con pHmetría esofágica. 
de bomba de protones, nos hubiera conducido a sobretratar a 3 pacientes.

Si bien es cierto NFL alterada y sugerente de RFL fue criterio de inclusión para nuestro estudio y por lo tanto todos los niños evaluados con sintomatología sospechosa de RFL como es la disfonía crónica, no todos tenían pH-IIM proximal compatible con RFL, por lo cual podemos decir, que no hay una clínica y NFL patognomónicos de RFL, como tampoco lo es la pHmetría alterada. Por otro lado, tanto la pHmetría normal por IR o por puntuación de DeMeester no descartan el RFL por lo que se requiere asociar IIM, lo que fue evidenciado por un índice de kappa aceptable, pero insuficiente. Publicaciones muestran que pHmetría convencional en el estudio sobretodo de síntomas extradigestivos de RGE, posee limitaciones tales como que identifica mal el reflujo de la columna completa y falla para correlacionar los síntomas con eventos de ácido esofágico, por lo que es una herramienta inadecuada para el diagnóstico de síntomas extraesofágicos ${ }^{20,21}$. Por otro lado, obtener datos en controles sanos no es éticamente viable debido a la naturaleza invasiva de la pHmetría, dificultando determinación de verdaderos valores "normales"16, con la desventaja, además que la pHmetría no detecta episodios de reflujo no ácidos. Por último, si bien la correlación de los síntomas con los eventos de reflujo es una de las indicaciones principales de la pHmetría, los pacientes y padres a menudo no informan los síntomas, un factor que compromete la correlación de los síntomas ${ }^{22}$. Además, se debate el marco temporal apropiado en el que considerar un síntoma correlacionado con el reflujo ${ }^{23}$.

En conclusión, no hay evidencia suficiente para respaldar el uso rutinario de pHmetría para el diagnóstico de enfermedad por RGE en pediatría.

En cuanto a Puntuación de DeMeester tiene la limitación de ser utilizado más rutinariamente en adultos que en niños. Los estudios realizados con pHmetría de 24 horas, basados en la puntuación de DeMeester, han mostrado tener una gran exactitud diagnóstica cuando la población que se estudia está constituida por pacientes con alta prevalencia de $\mathrm{RGE}^{17}$. A pesar de ello queremos hacer énfasis que en nuestro estudio pudimos identificar a 1 paciente que teniendo $\mathrm{pHmetría}$ normal, presentaba puntuación de DeMeester alterada y documentamos reflujo proximal por $\mathrm{pH}$-IIM.

El RFL puede presentarse tanto en lactantes como en niños pequeños, con diferentes síntomas dependiendo de la edad. En lactantes: disfagia, crup recurrente, estenosis subglótica o problemas respiratorios crónicos; niños escolarizados: tos crónica, disnea, disfonía, dolor de garganta persistente, halitosis y sensación de globo; niños mayores pueden referir problemas respiratorios crónicos ${ }^{4}$. No obstante, comparable con lo encontrado en nuestro estudio, se ha reportado que los síntomas de RFL son inespecíficos y el diagnóstico puede ser potencialmente confuso ${ }^{3,8}$. Esto, debido a que la mayoría de pacientes con RFL no muestran síntomas clásicos de enfermedad por RGE y en niños debido al patrón intermitente, incluso si se han observado síntomas tales como tos crónica, carraspera repetitiva con descarga retronasal, laringitis recurrente y espasmo laríngeo ${ }^{1}$.

Por otro lado, dentro de los hallazgos sugerentes en NFL se incluyen el eritema y edema de comisura posterior, edema de aritenoides, edema de la pared medial aritenoídea, granuloma de apófisis vocal, pseudo sulcus, pared posterior faríngea con aspecto de empedrado y edema de la pared posterior del cricoides ${ }^{1}$. De estos hallazgos el signo descrito con mayor frecuencia en pacientes con RGE, es el edema y eritema de la pared posterior de la faringe ${ }^{1}$, coincidiendo con los hallazgos comunes en pacientes con RFL en nuestro estudio, sin ser patognomónicos ${ }^{8}$.

Entre las limitaciones de este estudio destacan que la casuística fue muy pequeña, siendo por ende éste un reporte preliminar, la falta de grupo control, lo que no se consideró debido a las limitaciones éticas. Entre las fortalezas del estudio destaca su carácter prospectivo.

\section{Conclusiones}

En el 75\% de los pacientes con disfonía crónica y NFL sugerente de RFL se documentó reflujo proximal con pH-IIM, concluyendo que no todo paciente con disfonía y NFL sugerente de RFL lo tiene en realidad.

Sólo con la evaluación clínica, el estudio endoscópico flexible de la laringe y pHmetría tradicional no es posible confirmar diagnóstico de RFL, por lo que se recomienda asociar Impedanciometría a pHmetría para lograr mayor precisión diagnóstica de RFL, disminuyendo el sobrediagnóstico en el $25 \%$ de los casos.

\section{Responsabilidades Éticas}

Protección de personas y animales: Los autores declaran que los procedimientos seguidos se conformaron a las normas éticas del comité de experimentación humana responsable y de acuerdo con la Asociación Médica Mundial y la Declaración de Helsinki.

Confidencialidad de los datos: Los autores declaran que han seguido los protocolos de su centro de trabajo sobre la publicación de datos de pacientes.

Derecho a la privacidad y consentimiento informa- 
do: Los autores han obtenido el consentimiento informado de los pacientes y/o sujetos referidos en el artículo. Este documento obra en poder del autor de correspondencia.

\section{Conflicto de intereses}

Los autores declaran no tener conflicto de intereses.

\section{Agradecimientos}

Verónica Bley y Cecilia Gallo, Fonoaudiólogas y Verónica Lobos, Enfermera, Policlínico Otorrinolaringología, Hospital San Juan de Dios. Dra. Luisa Schonhaut, Investigadora. Pediatra. Clínica Alemana. Dra. Ana María Moraga, Epidemióloga, Departamento Educación Médica, Facultad de Medicina, Universidad de Concepción.

\section{Referencias}

1. Nazar R, Fullá J, Varas M, et al. Reflujo faringolaríngeo: Revisión bibliográfica. Universidad de Chile. Rev. Otorrinolaringol. Cir. Cabeza Cuello. 2008;68:193-8.

2. Galluzzi F, Schindler A, Gaini R, et al. The assessment of children with suspected laryngopharyngeal reflux: An Otorhinolaringological persepective. Int. J. Pediatr. Otorhinolaryngol. 2015;79:1613-9.

3. Cumpston E, Blumin J. and Bock J. Dual $\mathrm{pH}$ with Multichannel Intraluminal Impedance Testing in the Evaluation of Subjective Laryngopharyngeal Reflux Symptoms. Otolaryngol. Head Neck Surg. 2016;155(6):1014-20.

4. Venkatesan N, Pine H, Underbrink M. Laryngopharyngeal Reflux Disease in Children. Pediatric. Clin. N. Am. 2013;60:865-78.

5. Koufman J. Laryngopharyngeal Reflux 2002: A New Paradigm of Airway Disease. Ear Nose Throat J. 2002;81;2-6.

6. Lechien J, Finck C, Costa de Araujo P, et al. Voice outcomes of laryngopharyngeal reflux treatment: a systematic review of 1483 patients. Eur Arch Otorhinolaryngol. 2017;274:1-23.

7. Jiménez L, Mantilla N, Ospina J. Laringitis por reflujo: La perspectiva del otorrinolaringólogo. Rev. Col. Gastroenterol. 2011;26(3):198-206.

8. Ford C. Evaluation and management of Laryngopharyngeal Reflux. JAMA. 2005;294(12):1534-40.

9. Dulery C, Lechot A, Roman S, et al. A study with pharyngeal and esophageal 24-hour $\mathrm{pH}$-impedance monitoring in patients with laryngopharyngeal symptoms refractory to proton pump inhibitors. Neurogastroenterol. Motil. 2017;29:1-8.

10. Ozmen S, Demirceken F, Barut Y, et al. Role of laryngoscopy in children with respiratory complaints and suspected reflux. Allergol. Immunopath. 2012;40(4):204-9.

11. Orsi M, Cohen J, Donato G, et al. Estudio del Reflujo Gastroesofágico con Impedanciometría intraluminal multicanal en 160 niños. Abstracts: North American Society for Pediatric Gastroenterology, Hepatology, and Nutrition Annual Meeting October. 19-22,2006 Orlando, Florida. J Pediatr. Gastroenterol. Nutr. 2006;43:E14-E76.

12. Wenzl T, Benninga $M$, Loots $C$, et al. On Behalf of the ESPGHAN EUROPIG Working Group. Indications, Methodology, and Interpretation of Combined Esophageal Impedance-pH Monitoring in Children: ESPGHAN EURO-PIG Standard protocol. J Pediatr. Gastroenterol. Nutr. 2012;55:230-4.

13. Garza J, Nylund C and Kaul A. Time to stop blaming gastroesophageal reflux. Clin. Pediatr. 2011;50(12): 1110-5.

14. Olleta L, Cohen J, Orsi M. Laringitis recurrente de la infancia: Evaluación con impedanciometría Intraluminal Multicanal (IMM). Acta Gastroenterol Latinoam. 2013;43:9-11.

15. Patra S, Singh V, Chandra J. et al. Diagnostic modalities for gastroesophageal reflux in infantile wheezers. J. Trop. Pediatr. 2011;57:99-103.

16. Rosen R, Vandenplas Y, Singendonk M. et al. Pediatric Gastroesophageal Reflux Clinical Practice Guidelines: Joint Recommendations of the North American Society for Pediatric Gastroenterology, Hepatology, and Nutrition and the European Society for Pediatric Gastroenterology, Hepatology, and Nutrition. J. Pediatr. Gastroenterol. Nutr. 2018;66:516-54.

17. Canga F, Ciriza C, Ruiz de León A. Análisis de los resultados mediante puntuaciones compuestas. Relación entre datos de pHmetría y síntomas. En: Phmetría en la práctica clínica. Madrid: Editorial Ergon. 2019;53-4.

18. Landis J, Koch G. The measurement of observer agreement for categorical data. Biometrics. 1977;33:159-74.

19. Rosen R, Hart K, Nurko S. Does Reflux Monitoring with Multichannel Intraluminal Impedance Change Clinical Decision Making? J Pediatr. Gastroenterol. Nutr. 2011;52:404-7.

20. Chiou E, Rosen R, Jiang $\mathrm{H}$, et al. Diagnosis of supra-esophageal gastric reflux: correlation of oropharyngeal $\mathrm{pH}$ with esophageal impedance monitoring for gastro- esophageal reflux. Neurogastroenterol. Motil. 2011;23(8):717-e326.

21. Chiou E, Rosen R, Nurko S. Effect of different $\mathrm{pH}$ criteria on dual-sensor $\mathrm{pH}$ monitoring in the evaluation of supraesophageal gastric reflux in children. J. Pediatr. Gastroenterol. Nutr. 2011;52:399-403.

22. Rosen R, Amirault J, Giligan E, et al. Intraesophageal pressure recording improves the detection of cough during multichannel intraluminal impedance testing in children. J. Pediatr. Gastroenterol. Nutr.2014;58:22-6.

23. Farahmand F, Sabbaghian M, Ghodousi $\mathrm{S}$, et al. Gastroesophageal reflux disease and tooth erosion: a cross-sectional observational study. Gut. Liver. 2013;7:278-81. 